\title{
Design and Testing of the Strain Transducer for Measuring Deformations of Pipelines Operating in the Mining-deformable Ground Environment
}

\author{
Waclaw Gawedzki ${ }^{1}$, Jerzy Tarnowski ${ }^{2}$ \\ ${ }^{1}$ AGH University of Science and Technology, Department of Measurement and Electronics, al. Mickiewicza 30, \\ 30-059, Krakow, Poland, waga@agh.edu.pl \\ ${ }^{2}$ AGH University of Science and Technology, Department of Machines Design and Operation, al. Mickiewicza 30, \\ 30-059, Krakow, Poland, tarnow@agh.edu.pl
}

\begin{abstract}
Design and laboratory test results of the strain transducer intended for monitoring and assessing stress states of pipelines sited in mining areas are presented in this paper. This transducer allows measuring strains of pipelines subjected to external forces - being the mining operations effect. Pipeline strains can have a direct influence on a tightness loss and penetration of the transported fluid into the environment. The original strain gauge transducer was proposed for performing measurements of strains. It allows measuring circumferential strains and determining the value and direction of the main longitudinal strain. This strain is determined on the basis of measuring component longitudinal strains originating from axial forces and the resultant bending moment. The main purpose of investigations was the experimental verification of the possibility of applying the strain transducer for measuring strains of polyethylene pipelines. The obtained results of the transducer subjected to influences of tensile and compression forces are presented and tests of relaxation properties of polyethylene are performed.
\end{abstract}

Keywords: Strain transducer, pipeline, polyethylene, strain, stress, strain gauge.

\section{INTRODUCTION}

$\mathrm{P}$ IPELINES for transporting gases and liquids (e.g. gas pipelines, water supply systems, etc.) are made of steel or plastics (polyethylene, PCV). They are applied in dependence on the kind and pressure of transported media, area configurations and costs, at fulfilling conditions of the transport maximum safety and reliability. Pipelines utilised in mining-deformable ground environment can be subjected to additional deformations [1-4]. These areas are characterised by variable values of ground deformations reaching up to $9 \mathrm{~mm} / \mathrm{m}$ and above. They can have both a static and a dynamic character. On account of the above, materials applied for pipelines should compensate, as much as possible, constant and variable external loads [5 - 10]. They should also ensure self-compensation of plastic-elastic strains in the pipe material. Such special properties characterise polyethylene of a density higher than $930 \mathrm{~kg} / \mathrm{m}^{3}$, used in constructing pipelines. The expected servicing period of polyethylene pipelines equals approximately 50 years, when the maximal circumferential stresses are not higher than $8 \mathrm{MPa}$. In consideration of axial loads of the pipeline the most important parameters are: resistances to tension and compression of polyethylene pipes and the material self-relaxation time after the load withdrawal [9], [11].

The application of the strain gauge method for measuring pipeline strains has to take into account physical and mechanical properties of materials from which the pipeline is made [12-15]. A pipeline fragment constitutes an integral part of the measuring transducer. In measurements of strains it functions as an elastic element transferring stresses into strains. Out of that, the need of testing material properties from which the pipeline is built arises, since it is necessary for conclusions concerning the state of stresses in the pipeline on the bases of the measured strains. In case of steel structures these dependencies are well known, e.g. in [13] and [16] the strain measurement method and diagnostics of the steel pipeline was presented. However, in case of polyethylene the problems concern: nonlinearity and hysteresis of transferring characteristics, low values of the Young modulus of elasticity resulting in high strain values, long relaxation times, and possibilities of gluing strain gauges (only a few - from the accessible adhesives - are efficiently joining the polyethylene foundation with strain gauges) [6], [10], [17]. The results of the test performed, up to the present, concerning the mechanical properties of polyethylene, can be found in the references: its tightness, strength, relaxation time [6], [7], [9], [11], [18]. However, there is a lack of the results concerning measuring of polyethylene pipeline strains subjected to external loads with the application of the strain gauge measurement technique.

The theoretical bases of constructing the strain transducer for pipeline strains are presented in the following part of the paper. The results of laboratory tests of the strain transducer prototype for pipelines made of polyethylene are also shown.

\section{PRINCIPLE OF OPERATION OF THE STRAIN TRANSDUCER}

As the results of external loads of the pipeline, caused by an influence of unstable areas, the following can occur:

- components of longitudinal strains $\varepsilon_{l}$ caused by the axial force, and components of longitudinal strains $\varepsilon_{l b}$ caused by the resultant bending moment,

- circumferential strains $\varepsilon_{c}$,

- torsional strains. 
Torsional strains for load states occurring in rectilinear pipeline segments can be neglected, due to their small contribution into the total load balance [16]. The determination of strain components is necessary to determine the maximum longitudinal strains of the pipeline, which is in accordance with the direction of the resultant bending moment operation. The pipeline cross-section with the selected right-handed coordinate system $\mathrm{X}, \mathrm{Y}, \mathrm{Z}$, where axis $\mathrm{Z}$ is in accordance with the pipeline axis, is presented in Fig.1. [13].

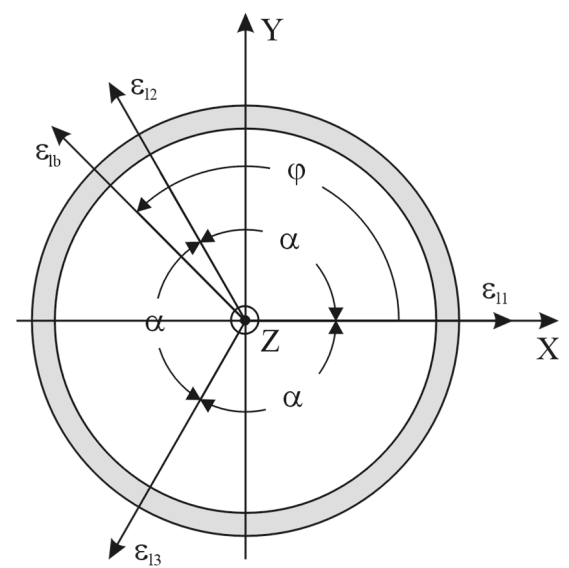

Fig.1. Placement of measuring points on the pipeline circumference for the 3-point method of strain measuring $(n=3$, $\alpha=120^{\circ}$ ).

Individual axes of the coordinate system determine the force directions. Axis $\mathrm{Z}$ determines the direction of the axial force action, while axes $\mathrm{X}$ and $\mathrm{Y}$ determine the radial direction of forces causing bending moments. Measuring axes $\varepsilon_{l 1}, \varepsilon_{l 2}, \ldots \varepsilon_{l n}$ were uniformly arranged, mutually at angle:

$$
\alpha=\frac{360^{\circ}}{n} \quad \text { for } \quad n \geq 3
$$

where: $n$ - number of measuring points on the tested pipeline circumference.

They determine - on the pipeline surface - the points of measuring complex longitudinal strains (along axis Z). For $n=3$ - there will be three measuring points distributed every $120^{\circ}$, for $n=4$ - four measuring points distributed every $90^{\circ}$, etc. Axis $\mathrm{X}$ was assumed as the reference axis, versus which the angle of the resultant bending moment $\varphi$ was determined. The first strain measuring axis $\varepsilon_{l 1}$ was defined along axis X. Longitudinal strain values $\varepsilon_{l X}, \varepsilon_{l Y}$ and $\varepsilon_{l Z}$ in the axes $\mathrm{X}, \mathrm{Y}$ and $\mathrm{Z}$ can be determined on the basis of equation [13]:

$$
\left[\begin{array}{c}
\varepsilon_{l X} \\
\varepsilon_{l Y} \\
\varepsilon_{l Z}
\end{array}\right]=\left(\mathbf{A}^{T} \cdot \mathbf{A}\right)^{-1} \cdot \mathbf{A}^{T} \cdot\left[\begin{array}{c}
\varepsilon_{l 1} \\
\varepsilon_{l 2} \\
\vdots \\
\varepsilon_{l n}
\end{array}\right]
$$

where: $\varepsilon_{l 1}, \varepsilon_{l 2} \ldots \varepsilon_{l n}$ - complex longitudinal strains measured in $n$ axes,

$\varepsilon_{l X}$ - longitudinal strain caused by the bending moment acting along axis $X$,

$\varepsilon_{l Y}$ - longitudinal strain caused by the bending moment acting along axis $\mathrm{Y}$,

$\varepsilon_{l Z}$ - mean longitudinal strain caused by axial force acting along axis $\mathrm{Z}$,

A - transformation matrix of dimensions $(n \times 3)$ in the form:

$$
\mathbf{A}=\left[\begin{array}{ccc}
-\cos (0 \cdot \alpha) & -\sin (0 \cdot \alpha) & 1 \\
-\cos (1 \cdot \alpha) & -\sin (1 \cdot \alpha) & 1 \\
\vdots & \vdots & \vdots \\
-\cos ((n-1) \cdot \alpha) & -\sin ((n-1) \cdot \alpha) & 1
\end{array}\right]
$$

In order to determine values of the strain components $\varepsilon_{l X}$, $\varepsilon_{l Y}$, and $\varepsilon_{l Z}$, measurements of complex strains should be performed, at least in three points on the pipeline circumference in the selected cross-section $\left(\varepsilon_{l 1}, \varepsilon_{l 2}\right.$ and $\varepsilon_{l 3}$ for $n=3$ ). The analysis carried out in paper [13] has shown that the smallest errors in determining components of longitudinal strains are obtained when four measuring axes are applied (strains measured every $\alpha=90^{\circ}$ on the pipeline circumference). It was also shown that, due to small differences in errors and saving the number of strain gauges and measuring channels, the measurement application in three axes is optimal. Each of the three strain gauges should be placed on the pipeline surface in the direction along the $\mathrm{Z}$ axis, in places determined by measuring axes $\varepsilon_{l 1}, \varepsilon_{l 2}$, and $\varepsilon_{l 3}$ [13], [16].

Equation (2) taking into account (3) for $n=3$ and $\alpha=120^{\circ}$ assumes the form:

$$
\left[\begin{array}{l}
\varepsilon_{l X} \\
\varepsilon_{l Y} \\
\varepsilon_{l Z}
\end{array}\right]=\left[\begin{array}{ccc}
\frac{2}{3} & -\frac{1}{3} & -\frac{1}{3} \\
0 & \frac{\sqrt{3}}{3} & -\frac{\sqrt{3}}{3} \\
\frac{1}{3} & \frac{1}{3} & \frac{1}{3}
\end{array}\right] \cdot\left[\begin{array}{c}
\varepsilon_{l 1} \\
\varepsilon_{l 2} \\
\varepsilon_{l 3}
\end{array}\right]
$$

Values of strain components can be determined on the basis of (4) from the dependence:

$$
\varepsilon_{l Z}=\frac{\varepsilon_{l 1}+\varepsilon_{l 2}+\varepsilon_{l 3}}{3}
$$

where: $\varepsilon_{l Z}$ - mean longitudinal strain (caused by the resultant force acting along axis $\mathrm{Z}$ ), and:

$$
\begin{gathered}
\varepsilon_{l b}=\sqrt{\varepsilon_{l X}^{2}+\varepsilon_{l Y}^{2}}= \\
=\frac{2}{3} \sqrt{\varepsilon_{l 1}^{2}+\varepsilon_{l 2}^{2}+\varepsilon_{l 3}^{2}-\varepsilon_{l 1} \varepsilon_{l 2}-\varepsilon_{l 1} \varepsilon_{l 3}-\varepsilon_{l 2} \varepsilon_{l 3}}
\end{gathered}
$$

where: $\varepsilon_{l b}$ - strain caused by the resultant bending moment.

On the basis of (4) angle $\varphi$ between axis $X$ and the direction of the resultant bending moment action can be determined: 


$$
\operatorname{tg} \varphi=\frac{\varepsilon_{l Y}}{\varepsilon_{l X}}=\frac{\sqrt{3}\left(\varepsilon_{l 2}-\varepsilon_{l 3}\right)}{2 \varepsilon_{l 1}-\varepsilon_{l 2}-\varepsilon_{l 3}}
$$

The extreme longitudinal strain $\varepsilon_{\text {lext }}$ will occur in the direction of the resultant bending moment action. It will reach the value equal to the sum of the mean longitudinal strain $\varepsilon_{l Z}$ (from axial forces (5)) and the component of longitudinal strain $\varepsilon_{l b}$ (from the resultant bending moment (6)), when both strains have the same sign [13], [16]:

$$
\varepsilon_{\text {lext }}=\varepsilon_{l Z}+\varepsilon_{l b}
$$

Stresses $\sigma$ caused by the axial force action $F_{Z}$ and by the bending moment $F_{b} \cdot l$ can be expressed by dependences:

$$
\sigma_{Z}=E \cdot \varepsilon_{l Z}=\frac{F_{Z}}{A} \quad \text { and } \quad \sigma_{b}=E \cdot \varepsilon_{l b}=\frac{F_{b} \cdot l}{w_{b}}
$$

where: $E$ - Young modulus of the pipe material, $A$ - surface of the pipe cross-section, $l$ - length of the bending force arm, $w_{b}$ - bending strength index.

Dividing equations (9) by members, we obtain:

$$
\frac{F_{Z}}{F_{b}}=\frac{\varepsilon_{l Z}}{\varepsilon_{l b}} \cdot \frac{A \cdot l}{w_{b}}
$$

Since the resultant force $F$ acting on the pipe equals:

$$
F^{2}=F_{Z}^{2}+F_{b}^{2}
$$

where: $F_{Z}$ - force acting along the pipeline,

$F_{b}$ - resulting bending force acting on the pipeline.

It is possible to determine component forces on the basis of the resultant force $F$ and strains $\varepsilon_{l Z}$ and $\varepsilon_{l b}$ at using dependences (10) and (11):

$$
F_{Z}=\frac{F}{\sqrt{1+\left(\frac{\varepsilon_{l b}}{\varepsilon_{l Z}} \cdot \frac{w_{b}}{A \cdot l}\right)^{2}}} \text { and } F_{b}=\frac{F}{\sqrt{1+\left(\frac{\varepsilon_{l Z}}{\varepsilon_{l b}} \cdot \frac{A \cdot l}{w_{b}}\right)^{2}}}
$$

The total value of longitudinal strains $\varepsilon_{l}$ will be the sum of these strains $\varepsilon_{\text {lext }}(8)$ caused by external forces and the internal pressure of the medium $\varepsilon_{l p}$ :

$$
\varepsilon_{l}=\varepsilon_{l \mathrm{ext}}+\varepsilon_{l p}
$$

An occurrence of longitudinal strains $\varepsilon_{l p}$ originating from the medium pressure will depend on the condition of the pipeline operation. If it is sited in the ground, friction forces can cause that longitudinal strains will not reveal themselves [16]. If the pressure in the pipeline is low (below $10 \mathrm{kPa}$ ), the effect of its influence can be either ignored or compensated. However, for medium-sized pressures (from $10 \mathrm{kPa}$ to $0.5 \mathrm{MPa}$ ) their influence should be compensated.
This problem is discussed more broadly in further part of the chapter.

Let us discuss the case of circumferential strains. The total value of circumferential strains $\varepsilon_{c}$ will be the sum of these strains caused by external forces $\varepsilon_{c e}$ and the internal pressure of the medium $\varepsilon_{\mathrm{c} p}$ :

$$
\varepsilon_{c}=\varepsilon_{c e}+\varepsilon_{c p}
$$

where: $\varepsilon_{\mathrm{c}}$ - total value of circumferential strains, $\varepsilon_{c e}$ - circumferential strains caused by external forces (axial forces and bending moment),

$\varepsilon_{c p}$ - circumferential strains being the effect of the pipeline stress $\sigma_{P}$ due to the medium pressure influence.

The maximal value of the circumferential strain $\varepsilon_{c \max }$ can be defined as the highest strain value measured in the circumferential direction:

$$
\varepsilon_{c \max }=\max _{i=1,2,3} \varepsilon_{c i}
$$

where: $\varepsilon_{c i}$ - circumferential strains measured in 3 points in the circumference ( $n=3$, in accordance with (1) and Fig.1.).

The pressure influence on the measurements of strain components, both longitudinal and circumferential, caused by external forces can be preliminarily compensated, e.g. by the proper balancing of the strain gauge bridge. However, such compensation method will not be efficient during a long-lasting monitoring and pressure changes of the medium. The efficient compensation can be then realised by the measurements of the actual value of pressure and determination of longitudinal $\varepsilon_{l p}$ and circumferential $\varepsilon_{c p}$ pipeline strains, caused by this pressure (adequate dependencies can be found in references, e.g. in [10]). For thin-walled pipe they take the form:

$$
\varepsilon_{l p}=\frac{p \cdot D}{4 \cdot t \cdot E}(1-2 v) \text { and } \varepsilon_{c p}=\frac{p \cdot D}{4 \cdot t \cdot E}(2-v)
$$

where: $p$ - pressure in the pipeline, $D$ - diameter of the pipe, $t$ - thickness of the pipe, $v$ - Poisson's ratio.

The influence of temperature changes on the measuring transducer can be compensated. To this aim, strain gauges intended for measuring strains in plastics (Fig.1.), operating in half-bridge systems, each with the compensating strain gauge, should be used.

In regard to the operations of the bridge with a single active strain gauge (second strain gauge in the half-bridge is passive and serves as the temperature change compensator) the nonlinearity error of the bridge will be shown. This error should be corrected in the case of measured big strain values $\varepsilon$. The equation for relative change of the bridge unbalance voltage $\Delta U / U_{s}$ has the form [13], [16]:

$$
\frac{\Delta U}{U_{s}}=\frac{1}{2} \cdot \frac{k \cdot \varepsilon+k \cdot\left(\varepsilon_{T}-\varepsilon_{T c}\right)}{2+k \cdot \varepsilon+k \cdot\left(\varepsilon_{T}+\varepsilon_{T c}\right)}
$$

where: $\Delta U$ - absolute change of the bridge unbalance voltage , $U_{s}$ - supply voltage of the bridge, $k$-strain gauge 
constant, $\varepsilon$ - measured strain, $\varepsilon_{T}, \varepsilon_{T c}$ - reaction of active and passive strain gauges for temperature variation in relation to the temperature of the system calibration.

To eliminate temperature effect, identical operational thermal condition for both strain gauges resulting in acceptance of $\varepsilon_{T} \approx \varepsilon_{T c}$ has to be secured. Because $\varepsilon_{T}$, and $\varepsilon_{T c}$ are much less than number 2 , then the equation (17) may be written in the form:

$$
\frac{\Delta U}{U_{s}}=\frac{1}{2} \cdot \frac{k \cdot \varepsilon}{2+k \cdot \varepsilon}
$$

The transformation equation of the bridge (18) is a nonlinear function with regard to $\varepsilon$. Its linear approximation takes the form:

$$
\left(\frac{\Delta U}{U_{s}}\right)_{l}=\frac{1}{4} \cdot k \cdot \varepsilon
$$

Then the relative nonlinearity error of the bridge with regard to (18) and (19) is:

$$
\delta_{n}=\frac{\left(\frac{\Delta U}{U_{s}}\right)_{l}-\frac{\Delta U}{U_{s}}}{\frac{\Delta U}{U_{s}}}=\frac{1}{2} \cdot k \cdot \varepsilon
$$

For small values of measured strain $\varepsilon$, e.g. steel pipes, when $\varepsilon \sim 1 \%$ and $k=2$, the relative nonlinearity error is $\delta_{n} \sim 0.1 \%$ and its neglecting may be considered. The strain value $\varepsilon$ may be determined from transformed linear relation (19).

For large values of measured strain $\varepsilon$, e.g. in the discussed case of polyethylene pipes, when $\varepsilon \sim 2 \%$ and $k=2$, the relative nonlinearity error is $\delta_{n} \sim 2 \%$ and most frequently it cannot be neglected. To eliminate the bridge nonlinearity error, the value of measured strain $\varepsilon$ should be determined on the base of the relation (18) transformed to the form:

$$
\varepsilon=\frac{4}{k} \cdot \frac{\Delta U}{U_{s}} \cdot \frac{1}{1-2 \cdot \frac{\Delta U}{U_{s}}}
$$

which is the function of measured relative value of the variation of the bridge unbalance voltage $\Delta U / U_{s}$.

\section{Purpose AND SUbJeCt OF LABORATORY TESTS OF THE TRANSDUCER}

The purpose of laboratory tests was the practical verification of properties of the strain transducer for the polyethylene pipeline and the determination of basic metrological characteristics. Due to the uniaxial strain distribution in the pipeline, caused by longitudinal forces, and by the resultant bending moment (algebraic sum strains), tests were performed for axial forces: tensile and compression.
Polyethylene PE-HD (high density PE) used for building gas pipelines has a density of $940-960 \mathrm{~kg} / \mathrm{m}^{3}$. It is obtained by low-pressure polymerisation. It is harder than polyethylene PE-LD (low density PE), has a higher mechanical strength and higher melting temperature $\left(125^{\circ} \mathrm{C}\right)$ [6], [7]. The most common trade brands of PE-HD are PE80 and PE100.

The segment of the typical pipe made of polyethylene PE100 of a coefficient SDR11 was the tested subject. Its external diameter was: $D=50 \mathrm{~mm}$ and wall thickness was $t=4.6 \mathrm{~mm}$. The basic physical and mechanical parameters are given in Table 1 .

Table 1. Basic physical and mechanical parameters of the polyethylene PE100 pipe (of the PipeLife Company).

\begin{tabular}{|l|l|}
\hline \multicolumn{1}{|c|}{ Parameter } & \multicolumn{1}{c|}{ Value } \\
\hline Young modulus of elasticity $E(1 \mathrm{~min})$. & $E \geq 1000 \mathrm{MPa}$ \\
\hline Yield strength & $R_{\mathrm{el}}=25 \mathrm{MPa}$ \\
\hline Elongation at the yield strength & $\varepsilon=9 \%$ \\
\hline Poisson ratio & $v=0.45$ \\
\hline
\end{tabular}

The strain transducer is presented in Fig.2.

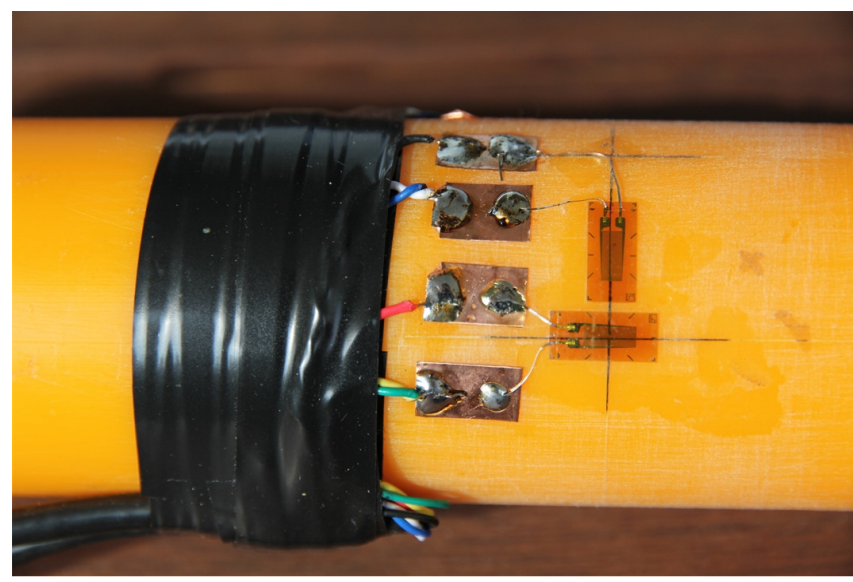

Fig.2. Strain transducer of the polyethylene pipeline strain measurements (one of three measuring points of longitudinal and circumferential strains).

Strain gauges of type 1-LD20-6/350 of the HBM Company [19] were used for measuring strains. They have an increased boundary value of measured strains to $10 \%$ (when the standard value is $5 \%$ ). Longitudinal and circumferential strains were measured in 3 points - situated uniformly on the pipe circumference $\left(n=3, \alpha=120^{\circ}\right)$ during the pipe compression and tension. Accuracy of the strain gauge constant is $\pm 1.5 \%$. Strain gauges were glued on the polyethylene pipe surface by means of the unary glue of ' $Z$ ' type [19]. Gauges cooperated with the amplifier Spider8 [19] of the accuracy class being $0.1 \%$. Bidirectional force sensor U9B (also of the HBM Company [19]) of a range of $20 \mathrm{kN}$ and accuracy $0.5 \%$ was applied for measuring the applied force. 


\section{LABORATORY TESTS AND THE OBTAINED RESULTS}

Within laboratory tests two experiments of compression [20] and tension of the strain transducer were performed. Both experiments were performed separately with two identically prepared transducers. In each test the transducer was mechanically connected in series with the pattern force sensor U9B and located in hydraulic press. In the first experiment the tension force was growingly set in linear mode with the use of the hydraulic press when in the second experiment the compression force was set in identical mode. In both experiments the press was shut off when the set force had been reached, and then the tested transducers were relieved. Values of applied forces were limited to the elasticity range of the material (Table 1.).

During experiments, the pipe strains as well as the applied resultant force $F(11)$ were measured as a time function. In order to determine relaxation properties of the polyethylene pipe, recording of strains was carried out also after finishing tension or compression. To eliminate errors caused by nonaxial operations of applied forces (either tension or compression), equations (5), (6) and (12) were used to determine the longitudinal force component $F_{Z}$. On the basis of strain $\varepsilon_{l i}(i=1 \div 3)$ and force $F$ measurements, for known values of structural parameters of the pipe, values of strain $\varepsilon_{l Z}$ and axial force $F_{Z}$ were determined - from these equations. Stresses $\sigma_{Z}$ - acting along the pipeline axis $Z$ were determined on the basis of equation (9). Tension and compression curves of the polyethylene pipe, obtained by axially acting force $F_{Z}$, are shown in Fig.3. Those curves were obtained from the determined time course of stress $\sigma_{Z}$ and the strain $\varepsilon_{l Z}$ as the result of time elimination.

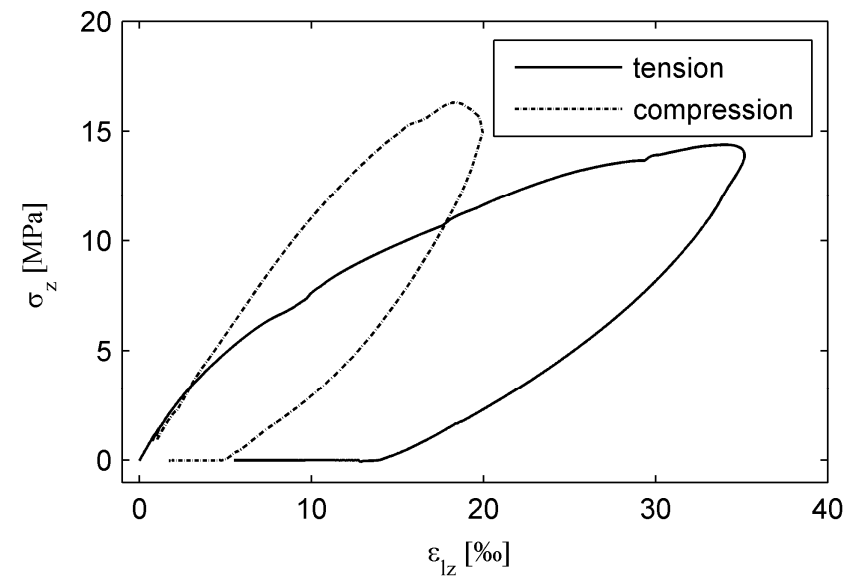

Fig.3. Tension and compression curves of the polyethylene PE100 pipe by axial force $F_{Z}$

On the basis of the obtained characteristics it can be stated that the value of the Young elasticity modulus decreases with the stress increasing. The polyethylene elastic element acts non-linearly. The obtained results confirm also the fact, that the polyethylene compression strength is higher than tensile strength (Young modulus decreases more slowly). A large hysteresis being the result of the long relaxation period of the material is also seen in diagrams (Fig.3.).
The effect of the polyethylene relaxation is best shown in Fig.4., in which the time-history of the longitudinal strain $\varepsilon_{l Z}$ is presented during tension and compression. The relaxation time of the pipe subjected to tension is longer than at compression. Apart from longitudinal strains also circumferential ones caused by the longitudinal force were measured in the tested transducer. Diagrams showing the dependence of longitudinal strains $\varepsilon_{l 1}$ on circumferential $\varepsilon_{c 1}$, are presented in Fig.5.

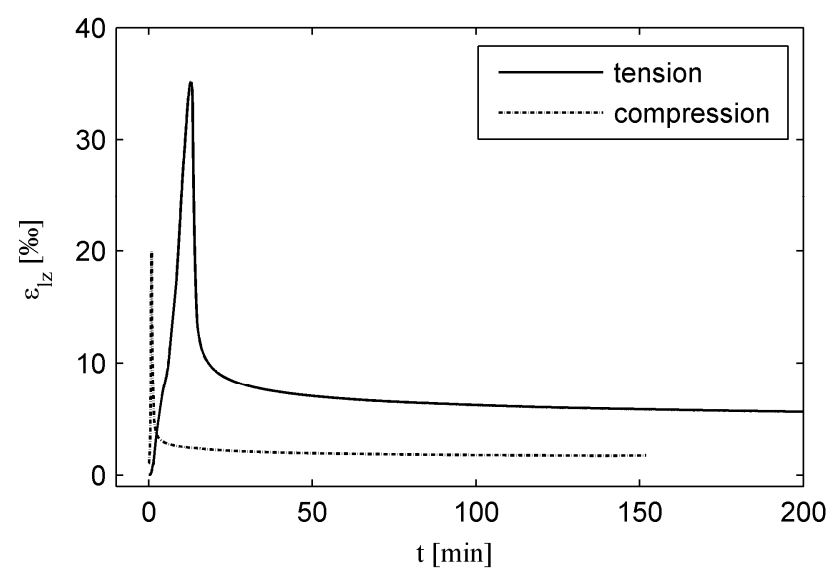

Fig.4. Time-history of the tension and compression processes and the polyethylene pipe relaxation.

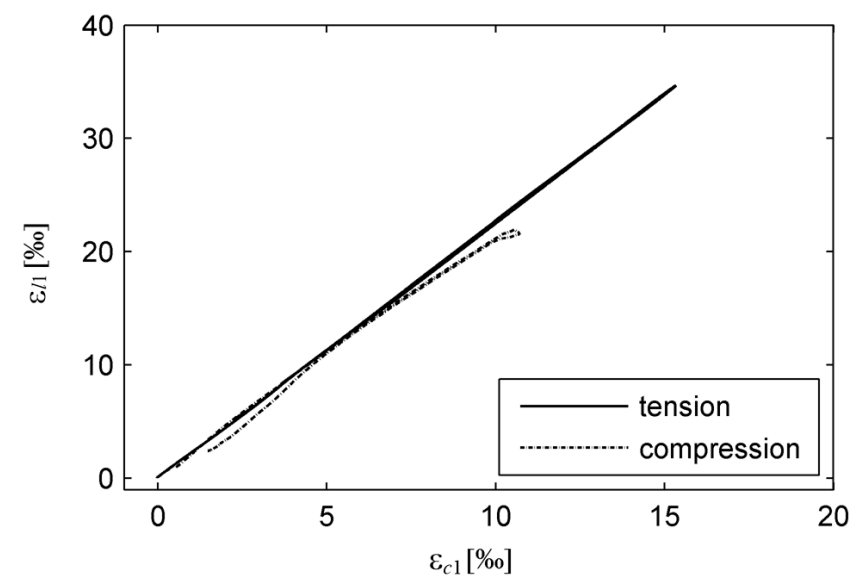

Fig.5. Dependence of the longitudinal strain gauge $\varepsilon_{l 1}$ strain as a function of the strain of the circumferential strain gauge $\varepsilon_{c 1}$, during tension and compression tests.

These strains were measured by means of the pair of mutually perpendicular strain gauges - glued in the selected measuring point (Fig.2.) - during tension and compression forces operations. They show a good accordance of both characteristics in the tested range. The Poisson ratio value determined on their bases equals: $v=0.43$ and is, within measuring errors and a natural scatter of material parameters, in agreement with the rated value given in Table 1. 


\section{TRANSDUCER APPLICATION IN PIPELINE DIAGNOSTICS}

The results of investigations presented in this paper constitute an essential contribution into the diagnostics and estimations of the maintenance state of pipelines made of plastics. This concerns also low- and middle-pressure polyethylene gas grids, currently widely applied. These problems are especially essential from the point of view of gas pipeline maintenance, which constitute threats for people.

Gas pipelines operating in mining areas are endangered by loads caused by area deformations. These area deformations cause pipeline strains which can lead to loosing tightness. Long-lasting loads can cause changes in wall thickness of gas pipelines and gas can permeate through them. In addition, the problems listed below can occur.

- Material fatigue of a pipe wall causes that as the time passes the resistance to pressure of the polyethylene pipelines decreases, due to the so-called stress corrosion [8].

- Material flowing due to large loads causes that the pipe is endangered by the phenomenon of propagation of cracks [10].

The strain transducer proposed in the paper allows controlling the pipeline state stress and can be applied for the estimation of the maintenance state and polyethylene pipeline tightness.

The method of the tightness testing of pipelines made of plastics, presented in [11], did not take into account monitoring of external load influences on pipelines. Characteristics of polyethylene, showing the dependence of the Young modulus reciprocal $(1 / E)$ as a function of time (expressed in the logarithmic scale) of the long-lasting pipeline load has - in approximation - a linear character (after a certain time from the beginning of load) [11]. Such type of dependency means that at the assurance (during the tightness test) of the determined and invariable stress $\sigma$ in the polyethylene pipeline (also $\sigma_{p}, \sigma_{z}$ and $\sigma_{b}(9)$ ) its strain increases as time passes. This condition will be met at maintaining the constant medium pressure and the constant external load during the tightness test of some hours. The applied tightness criterion [11] assumes measuring the gas volume with which the pipeline should be gradually supplemented - due to changes of the pipeline volume - to maintain the constant pressure during the whole tightness test. It is assumed that the constant pressure influence constitutes the source of volume changes of the tested pipeline fragment. Measurements of circumferential and longitudinal pipeline strains will allow determining actual volume changes of the pipeline not only due to the gas internal pressure but also due to external forces and moments acting on the pipeline. This is especially important during tightness tests of pipelines operating in unstable mining-deformable grounds. The modified tightness criterion can be then formulated.

In the case of a long pipeline the application of a strain gauge transducer in testing of the deformation and tightness condition may be limited. In such situation the measurement points should be more concentrated in parts of pipeline subjected to deformation.

\section{CONCLUSION}

The method of monitoring strain states of pipelines situated in unstable mining areas - by means of the strain transducer, is proposed in this paper. Innovations of the method are:

- application of the strain gauge measurement technique for measuring strains of polyethylene pipelines, currently used for building gas pipelines,

- constructing the prototype strain transducer for polyethylene pipelines and performing laboratory tests.

The determined transformation characteristics allowed the verification of the transducer metrological properties. They confirmed the possibility of application of the strain gauge method for measuring strains of polyethylene pipelines. However, due to non-linearity and hysteresis of stress characteristics (Fig.3.), possibilities of direct concluding on strain levels on the basis of measured strains are significantly limited.

A long relaxation time of polyethylene does not limit the transducer measuring possibilities in the range of quasistatic strains, but limits in the range of dynamic strains.

The possibility of the proper gluing of strain gauges on the polyethylene surface was positively verified.

The results presented in the paper constitute the grounds for concluding that strain measurements can be also useful in assessing the tightness of gas pipelines of polyethylene operating in mining-deformable grounds. Further investigations will concern assessments of how fatigue of the polyethylene pipeline wall, subjected to long-lasting variable loads, influences maintaining the pipeline tightness.

\section{REFERENCES}

[1] Abdoun, T.H., et al. (2009). Factors influencing the behavior of buried pipelines subjected to earthquake faulting. Soil Dynamics and Earthquake Engineering, 29 (3), 415-427.

[2] Honegger, D.G., Wijewickreme, D. (2013). Seismic risk assessment for oil and gas pipelines. In Seismic Risk Analysis and Management of Civil Infrastructure Systems. Woodhead Publishing, Part IV, 682-715.

[3] Lanzano, G., et al. (2014). Seismic vulnerability of gas and liquid buried pipelines. Journal of Loss Prevention in the Process Industries, 28, 72-78.

[4] Chen, W.W., et al. (2002). Seismic response of natural gas and water pipelines in the Ji-Ji earthquake. Soil Dynamics and Earthquake Engineering, 22 (9-12), 1209-1214.

[5] Figiel, W., Tarnowski, J. (2005). Badania wspolczynnika tarcia rurociągu i gruntu na terenach gorniczych w obecnosci wstrzasow. Eksploatacja $i$ Niezawodnosc-Maintenance and Reliability, 4, 55-63.

[6] Khan, F., Yeakle, C., Gomaa, S. (2012). Characterization of the mechanical properties of a new grade of ultra high molecular weight polyethylene and modeling with the viscoplasticity based on overstress. Journal of the Mechanical Behavior of Biomedical Materials, 6, 174-180. 
[7] Kiass, N., Khelif, R., Boulanouar, L., Chaoui, K. (2005). Experimental approach to mechanical property variability through a high-density polyethylene gas pipe wall. Journal of Applied Polymer Science, 97, 272-281.

[8] Zheng, W., Elboujdaini, M., Revie, R.W. (2011) Stress corrosion cracking in pipelines. In Stress Corrosion Cracking - Theory and Practice. Woodhead Publishing, 749-771.

[9] Bai, Q., Bai, Y. (2014). Subsea Pipeline Design, Analysis, and Installation (1st ed.). Elsevier.

[10] Inaudi, D. (2013). Pipelines. In Handbook of Technical Diagnostics. Fundamentals and Applications to Structures and Systems. Springer, 453-470.

[11] Janson, L.E. (1993). Method for tightness testing of plastics pressure pipelines. Construction and Building Materials, 7 (4), 241-244.

[12] Frings, J., Walk, T. (2011). Distributed fiber optic sensing enhances pipeline safety and security. Oil Gas European Magazine, 37 (3), 132-136.

[13] Gawedzki, W. (2001). Optymalizacja tensometrycznego czujnika odkształcen gazociagu eksploatowanego w warunkach niestabilnego srodowiska. In Materiaty Kongresu Metrologii KKM, 24-27 June 2001. Warszawa, Poland: BEL Studio, 2, 605-608.

[14] Gawedzki, W., Jurkiewicz, J. (1996). A branched system for measurements of the slow-variable forces using a strain gauge method. In IEEE Instrumentation and Measurement Technology Conference \& IMEKO Technical Committee 7 (IMTC/96 - IMEKO TC-7), 4-6 June 1996, Brussels, Belgium. IEEE, Vol. I and II, 196-199.
[15] Walk, T., Frings, J. (2010). Fiber optic sensing can help reduce third-party threats. Oil \& Gas Journal, 108 (33), 128-133.

[16] Hofstötter, P., Kosiedowski, P. (1971). Messung der durch Erdabsenkungen hervorgerufenen Zusatzbeanspruchungen an erdverlegten Fernleitungen. Sonderdruck aus Bänder Bleche Rohre, (12) 6, 261-265.

[17] Reed, Ch., Robinson, A.J., Smart, D. (2004). Techniques for monitoring structural behaviour of pipeline systems. Amer Water Works Assn.

[18] Qingmin, H., et al. (2013). Natural gas pipeline leakage detection based on FBG strain sensor measuring technology and mechatronics automation (ICMTMA). In Proceedings of Fifth IEEE International Conference on Measuring Technology and Mechatronics Automation, 16-17 January 2013. IEEE, 712-715.

[19] HBM Test and Measurement. http://www.hbm.com/.

[20] Syrek, K. (2013). Laboratoryjna weryfikacja tensometrycznej metody pomiaru odkształceń polietylenowych rur gazowych. Unpublished B.Sc. Thesis, AGH-UST Krakow, Poland.
Received January 15, 2015. Accepted October 07, 2015. 